\title{
Distribución espacial de Vanilla bahiana (Orchidaceae) en dos fitofisonomías de restinga. ¿El patrón espacial varía?
}

\author{
FELIPE FAJARDO VILLELA ANTOLIN BARBERENA ${ }^{1,2}$, ESTÍBALIZ LÓPEZ \\ HERMOSO $^{3,4} \&$ MARIA APARECIDA JOSÉ DE OLIVEIRA ${ }^{4}$
}

\author{
${ }^{1}$ Universidade Federal Rural da Amazônia, campus Capitão Poço, Herbário HCP, Estrada do Pau Amarelo s.n., \\ Vila Nova, BR-68650-000 Capitão Poço, Pará, Brasil \\ ${ }^{2}$ Museu Paraense Emílio Goeldi, Coordenação de Botânica, av. Perimetral 1901, Terra Firme, BR-66077-830 Belém, \\ Pará, Brasil \\ ${ }^{3}$ Universidad de Alcalá, Plaza de San Diego, s/n, ES-28801 Alcalá de Henares, Madrid, España \\ ${ }^{4}$ Universidade Federal da Bahia, Instituto de Biologia, Barão de Geremoabo 147, Ondina, BR-40170-290 Salvador, \\ Bahia, Brasil
}

ORCID iD. F. F. V. A. Barberena: http://orcid.org/0000-0002-7298-2977, E. López Hermoso: http://orcid.org/0000-0002-8039-5959, M. A. J. de Oliveira: http://orcid.org/0000-0002-8863-8951

Autor para correspondencia: F. F. V. A. Barberena (felipe.fajardo@ufra.edu.br)

Editores: J. López-Pujol \& N. Nualart

Recibido 31 agosto 2020; aceptado 5 diciembre 2020; publicado on line 30 abril 2021

\begin{abstract}
Spatial distribution of VANILLA BAHIANA (OrChidACEAE) IN TWO RESTINGA PHYTOPHYSIOGNOMIES. DOES THE SPATIAL PATTERN VARY? - Vanilla bahiana is an orchid restricted to Brazil, extremely ornamental, threatened with extinction and with economic potential. We evaluated the spatial distribution pattern of $V$. bahiana in shrub and restinga forest formations in a conservation unit in Northeast Brazil. The Morisita's index of dispersion, the Morisita's standardized index of dispersion and the TTLQV method were used, and $1920 \mathrm{~m}^{2}$ were sampled, through the demarcation of six transects of $40 \times 4 \mathrm{~m}$ per phytophysionomy. Each transect was subdivided into 10 plots of $4 \times 4 \mathrm{~m}$. In the shrub formation, we observed 49 individuals of $V$. bahiana and a greater abundance at the edges of the transects (plots 1 and 2). In the restinga forest, we recognized 47 individuals of $V$. bahiana and a distribution more homogeneous way along the transects. We found an aggregate pattern for the species in the shrub formation and a random pattern in the restinga forest. The different patterns could be explained by differences in luminosity, but they could also be associated with the dispersal of seeds or the presence of fungi of different species. Future studies with spatial distribution in Orchidaceae should be multidisciplinary in order to investigate the main causes of the patterns on local scales. Specifically, understanding the relationships between Vanilla species and mycorrhizal fungi may have positive impacts on the cultivation and commercialization of vanilla.
\end{abstract}

Key words: Brazil; conservation; ecology; microdistribution; orchids; vanilla.

\section{Resumen}

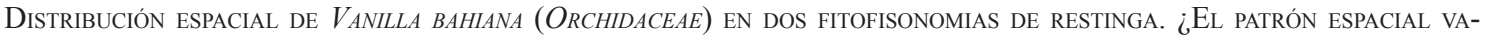
RíA? - Vanilla bahiana es una orquídea hemiepífita restringida a Brasil, extremadamente ornamental, amenazada de extinción y con potencial económico. Se evaluó el patrón de distribución espacial de $V$. bahiana en las fitofisionomías arbustiva y de bosque en un fragmento de llanuras costeras (restinga), en una unidad de conservación en el Noreste de Brasil. Para ello, se utilizó el índice de Morisita, el índice de Morisita estandarizado y el método TTLQV, y se muestrearon $1920 \mathrm{~m}^{2}$, a través de la demarcación de seis transectos de $40 \times 4 \mathrm{~m}$ por fitofisionomía. Cada transecto se subdividió en 10 parcelas de $4 \times 4 \mathrm{~m}$. En la formación arbustiva, se observaron 49 individuos of $V$. bahiana y mayor abundancia en los bordes de los transectos (parcelas 1 y 2). En el bosque de restinga se contaron 47 individuos of $V$. bahiana, pero distribuidos de manera más homogéna en los transectos. Se encontró un patrón agregado para la especie en la formación arbustiva y un patrón aleatorio en el bosque de restinga. Los diferentes patrones podrían explicarse por las diferencias de luminosidad, pero también podrían estar asociados con la dispersión de semillas o la presencia de 
hongos de diferentes especies. Futuros estudios con distribución espacial de Orchidaceae deberían ser pluridisciplinares con la finalidad de investigar las causas principales de los patrones en escalas locales. Específicamente, comprender las relaciones entre especies de Vanilla y los hongos micorrícicos puede generar impactos positivos sobre el cultivo y la comercialización de vainilla.

Palabras clave: Brasil; conservación; ecología; microdistribución; orquídeas; vainilla.

\section{Cómo citar este artículo / Citation}

Barberena, F. F. V. A., López Hermoso, E. \& Oliveira, M. A. J. de. 2021. Distribución espacial de Vanilla bahiana (Orchidaceae) en dos fitofisonomías de restinga. ¿El patrón espacial varía? Collectanea Botanica 40: e001. https://doi. org/10.3989/collectbot.2021.v40.001

\section{Copyright}

(C) 2021 CSIC. This is an open-access article distributed under the terms of the Creative Commons Attribution 4.0 International (CC BY 4.0) License.

\section{INTRODUCCIÓN}

Los patrones de distribución espacial de los organismos se pueden estudiar a diferentes niveles, desde el nivel de región biogeográfica hasta el de la población (Hay et al., 2000; Tremblay et al., 2006). La distribución espacial en microescala puede ser aleatoria, regular o agrupada y es determinada por factores abióticos (p. ej. disponibilidad de agua, luz y nutrientes; temperatura y humedad adecuadas) y bióticos (p. ej. competencia y herbivoría), que interactúan entre sí, y son influenciados por la acción antrópica y estocasticidad (Hutchings, 1997). La información sobre patrones espaciales puede contribuir a una mejor comprensión de los procesos de dispersión de especies y recolonización de hábitats, así como para el establecimiento de estrategias de conservación y planes de gestión, especialmente aquellos que involucren la recuperación de áreas degradadas o fragmentos de vegetación (Anjos et al., 2004; Bonet et al., 2005). Se han realizado evaluaciones del patrón de distribución espacial para especies de diferentes familias de angiospermas, helechos y gimnospermas, presentes en dominios fitogeográficos o biomas diversos en diferentes etapas sucesionales (Hay et al., 2000; Anjos et al., 2004; Kanieski et al., 2012; Sousa \& Cunha, 2018; Teixeira et al., 2019). En este sentido, las Orchidaceae han sido uno de los grupos botánicos más investigados; los estudios se centran en especies terrestres y tipos de vegetación del bosque atlántico, como el bosque caducifolio estacional, el bosque semideciduo estacional y llanuras costeras (restingas) (Budke et al., 2004; Krahl et al., 2010; Carvalho et al., 2012; Souza et al., 2012; Dubbern et al., 2013; Piccoli et al., 2014; Quenzer \& Pedroso-de-Moraes, 2014;
Souza-Leal \& Pedroso-de-Moraes, 2014; Fajardo et al., 2015; Rahal et al., 2015).

Los mecanismos de dispersión de semillas interfieren primariamente en el patrón espacial y son corresponsables de los niveles de diversidad genética en Orchidaceae (Chung \& Chung, 1999; Jacquemyn et al., 2007). Sin embargo, otros factores también influyen en la distribución de las poblaciones de orquídeas, como las condiciones de microclima, principalmente humedad relativa, temperatura e intensidad de luz; el paisaje en sí; las características morfológicas de la semilla; los sitios de dispersión de propágulos; y la relación con hongos micorrícicos (Budke et al., 2004; Phillips et al., 2010; Piccoli et al., 2014; Rahal et al., 2015). Las especies de orquídeas son particularmente vulnerables a la extinción, ya que, entre otros factores, con frecuencia tienen poblaciones reducidas, con una distribución restringida, y dependen al menos parcialmente de los hongos, principalmente, con respecto a la germinación y el desarrollo de plántulas (Batty et al., 2001; Barros, 2007; Pandey et al., 2013). Además, están sujetas a colecciones depredadoras por coleccionistas y comerciantes, que alimentan el comercio ilegal activo (Barros et al., 2007; Barberena et al., 2018).

En Brasil, un país megadiverso en especies de plantas, las restingas han sufrido con la retirada clandestina de arena, colección depredadora de especies nativas, deforestación y la expansión inmobiliaria (Britto et al., 1993; Souza et al., 2012). Vanilla bahiana Hoehne es una orquídea restringida a Brasil, que presenta un elevado interés ornamental y se encuentra en los dominios fitogeográficos de la Caatinga, Cerrado y el Bosque Atlántico, donde habitan principalmente en las restingas (Ferreira et al., 2017; Barberena et al., 2019; Flora do Brasil, 
2020). La especie es indicadora de la etapa avanzada de regeneración en fitofisiognomías arbustivas y de bosque de restinga en el estado de Bahía (MMA, 2012).

Recientemente, se ha sugerido el uso de V. bahia$n a$ en los esfuerzos de mejora genética del género Vanilla Mill., ya que la especie es filogenéticamente cercana a Vanilla planifolia Jacks. ex Andrews, la vainilla más importante económicamente, y tiene varios atributos ecológicos deseables, como gran cantidad de flores por racimo, extenso período de floración, fructificación durante todo el año y alta resistencia al estrés abiótico (Bouetard et al., 2010; Anjos et al., 2017; Nascimento et al., 2019). Además, $V$. bahiana produce cantidades significativas de vainillina, sustancia extraída de los frutos maduros y que es ampliamente utilizada en la gastronomía mundial e incluso en medicina (Bythrow, 2005; Lopes et al., 2019). Sin embargo, $V$. bahiana ha sido evaluada como en peligro de extinctión dado que la mayoría de las poblaciones se encuentran en hábitats muy fragmentados (Ferreira et al., 2017). El conocimiento sobre distribuciones en microescalas y particularidades ecológicas de parientes silvestres de la vainilla es deficiente, incluso sobre $V$. bahiana, y necesario para que futuras acciones de conservación y programas de cultivo sostenible se establezcan de manera más adecuada (Nascimento et al., 2019; Watteyn et al., 2020).

En ese contexto ecológico-conservacionista y considerando también el potencial económico de la especie, el presente estudio tiene como objetivo evaluar el patrón de distribución geográfica de $V$. bahiana en dos fitofisionomías de restinga en una unidad de conservación en el estado de Bahía. En particular, buscamos responder ¿El patrón espacial de una especie varía en fitofisionomías diferentes del mismo tipo de vegetación, en la misma zona?

\section{MATERIAL Y MÉTODOS}

\section{Área de estudio}

El estudio se realizó en el Área de Protección Ambiental de Lagunas y Dunas de Abaeté (APA Abaeté), que comprende $c a .1800$ hectáreas y está ubicado en el municipio de Salvador, Bahía, Brasil
(Diário Oficial do Estado da Bahia, 1987). El clima de la región se clasifica como Af, definido como cálido y húmedo, sin estación seca (Alvares et al., 2013). La temperatura media anual y la precipitación media anual en la región superan $\operatorname{los} 25^{\circ} \mathrm{C}$ y $2000 \mathrm{~mm}$, respectivamente, y la humedad relativa varía entre $70-80 \%$ durante la mayor parte del año (Viana \& Kleinert, 2005). Las elevaciones del terreno no superan los $100 \mathrm{~m}$. El APA Abaeté cubre dos parques municipales, el Parque do Abaeté, con 270 ha, y el Parque Municipal das Dunas, con 290 ha, que es el fragmento de vegetación mejor conservado de la región (Diário Oficial do Município de Salvador, 2011; Fig. 1).

Hasta ahora, las fitofisionomías del APA Abaeté no se han categorizado formalmente, pero las formaciones Arbusto no Inundable (AR) y Bosque de Restinga no Inundable (BR) son fácilmente reconocibles y se han adoptado en el presente estudio. La formación Arbusto no Inundable se encuentra comúnmente en lugares ligeramente más altos, sin inundaciones periódicas o constantes, y consiste en arbustos mezclados con bromelias terrestres y cactos que forman matorrales de diferentes extensiones y alturas, intercalados con extensas áreas abiertas de arena, el principal constituyente del sustrato en estas formaciones. La formación BR también es libre de inundaciones periódicas por la subida del nivel freático o el desborde de lagunas perennes y se puede caracterizar por un dosel de hasta $15 \mathrm{~m}$ y una capa evidente de materia orgánica sobre el suelo. Attalea funifera Mart. (Arecaceae) es una de las especies más características de esta formación. Datos fitofisiográficos adicionales se pueden obtener de Barberena et al. (2019).

\section{Breve caracterización morfológica y ecológica de la especie}

Vanilla bahiana se identifica fácilmente en la zona por el hábito hemiepífito (germina en el suelo, pero busca un soporte sobre el cual crece y eventualmente pierde el contacto con el suelo), pero también por un conjunto de características morfológicas: entrenudos espaciados (4 $\mathrm{cm}$ o más); hojas sésiles y oblongas; sépalos y pétalos verdes; y labelo blanco, entero, con franjas amarillas longitudinales y margen amarillo verdoso a amarillo (Fig. 2). La especie es autocompatible, aunque depende de las abejas del género Eulaema Lepeletier, 


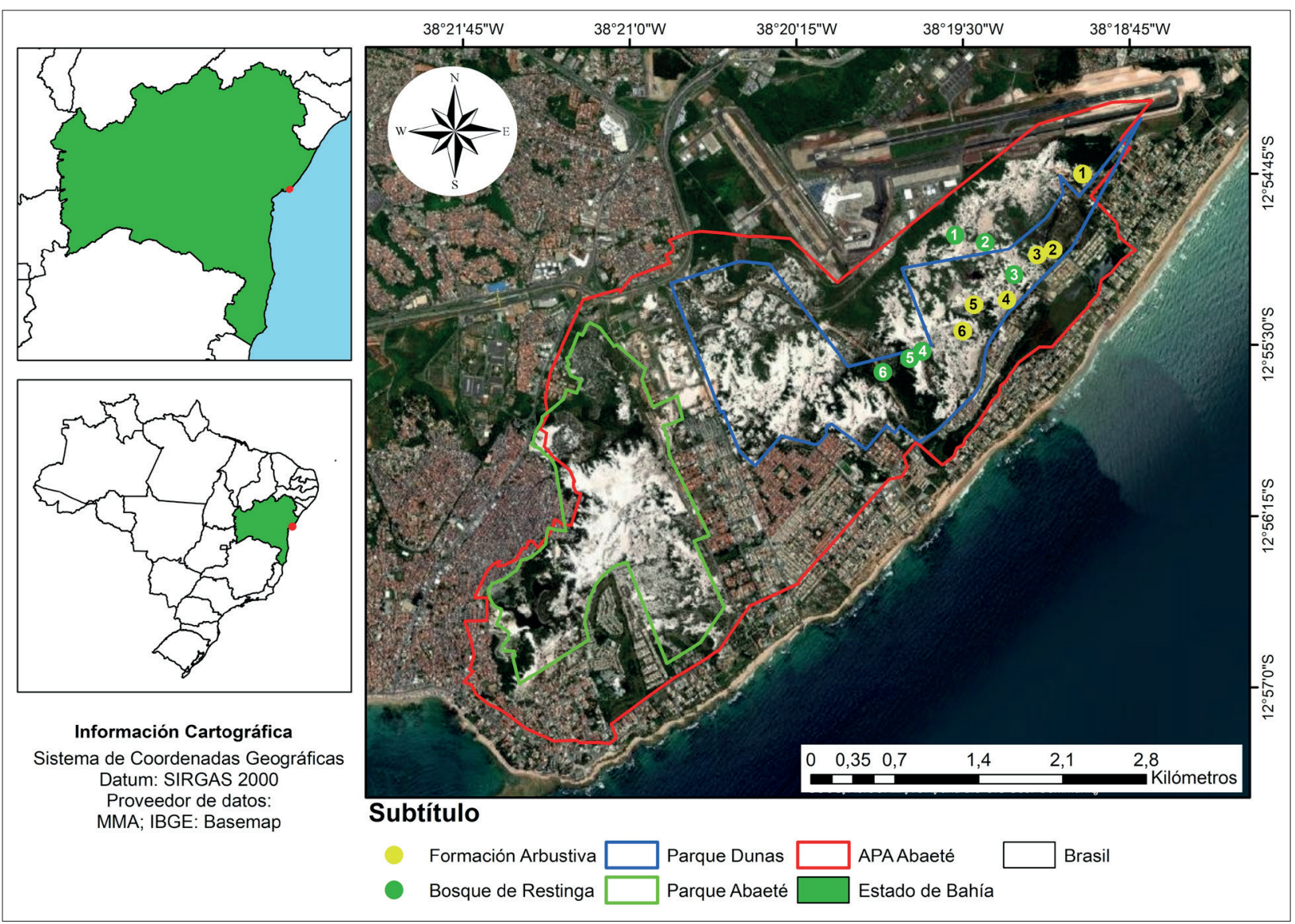

Figura 1. Área de Protección Ambiental de las Lagunas y Dunas de Abaeté, Bahía, Brasil, destacando el Parque Municipal Abaeté y el Parque Municipal Dunas. Los puntos amarillos y los puntos verdes corresponden, respectivamente, a los transectos establecidos en la formación arbustiva y en el bosque de restinga para evaluar la distribución espacial de $V$. bahiana (mapa: José Antônio Lima Rocha Junior).

1841 (Apidae, Euglossinae) para su polinización y reproducción, y presenta tasas bajas de fructificación en la restinga (Anjos et al., 2017). Los individuos florecen y dan frutos durante la mayor parte del año y muestran un crecimiento exacerbado en la unidad de conservación, llegando a decenas de metros, aunque las partes vegetativas en contacto directo con el suelo y expuestas al sol están claramente dañadas (Anjos et al., 2017; Barberena et al., 2019).

\section{Recolección de datos}

Entre noviembre de 2014 y marzo de 2015, dos investigadores cuantificaron y recuantificaron los individuos de $V$. bahiana de forma independiente, para evitar cualquier divergencia numérica (Barberena et al., 2019). Se recolectaron y se herborizaron dos especímenes (Barberena et al.
331; Barberena \& Aguiar 334) de acuerdo con las técnicas taxonómicas habituales y se depositaron en el herbario ALCB (Peixoto \& Maia, 2013). Se muestreó un área total de $1920 \mathrm{~m}^{2}$, mediante el establecimiento de doce transectos de $40 \times 4 \mathrm{~m}$, seis en la formación de arbustos no inundable y otros seis en la formación de bosque no inundable (Fig. 1). Los transectos fueron implantados en el Parque Municipal das Dunas, la porción más conservada de APA Abaeté, y sus alrededores (Fig. 1), con el fin de minimizar posibles interferencias en el análisis de los resultados, tales como la recolección depredadora de orquídeas y los efectos asociados con la fragmentación de la vegetación. Se utilizaron fragmentos grandes para contener 10 parcelas de $4 \times 4 \mathrm{~m}$ separados a una distancia mínima de 50 m entre sí, para evitar superposición. Sobre la base de estas premisas, el sistema de selección de las unidades de muestreo fue mixto, con la selección 

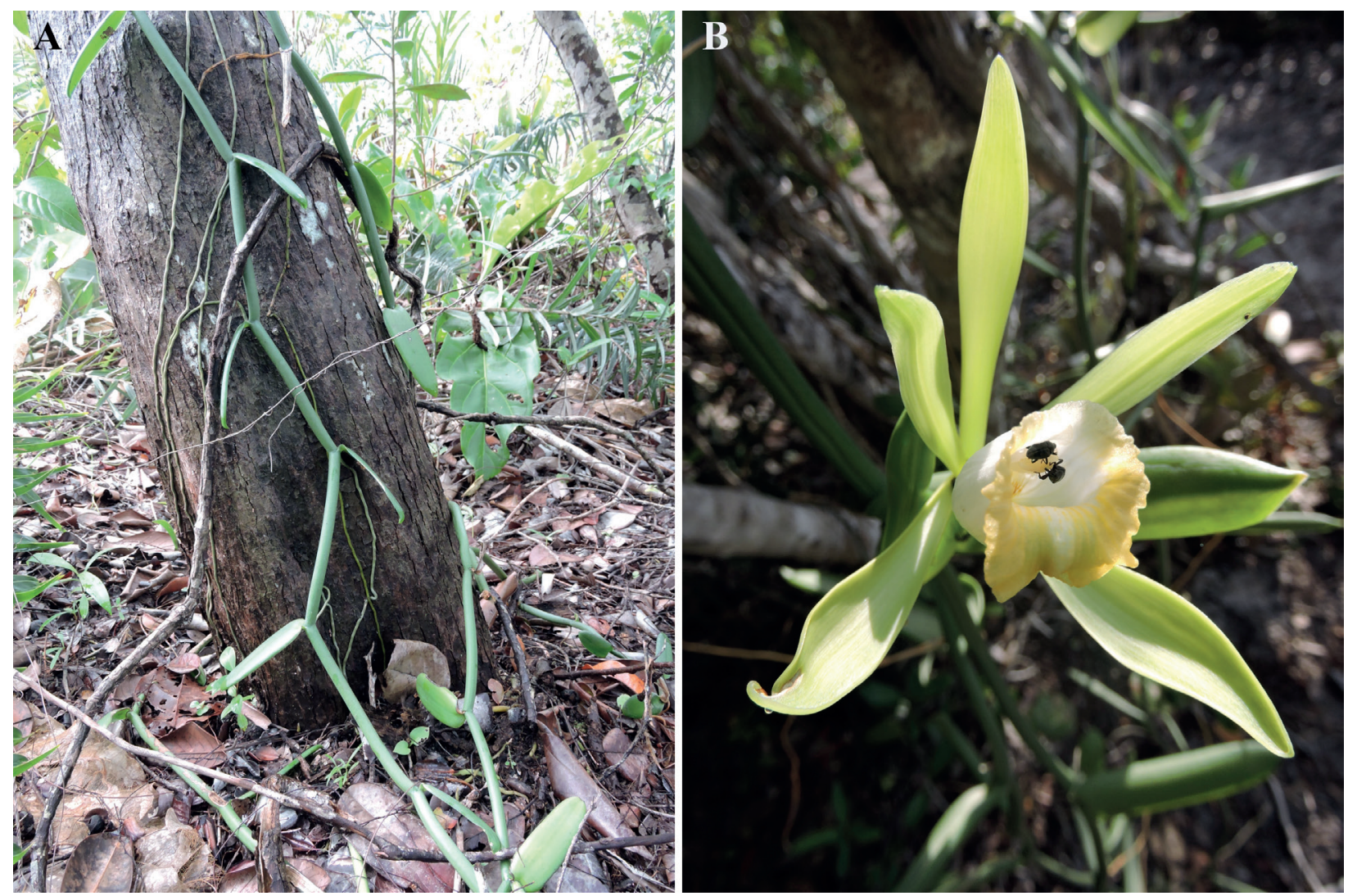

Figura 2. Vanilla bahiana: (A), hábito hemiepífito; (B), flor en vista frontal (fotografías: Felipe Fajardo Villela Antolin Barberena).

aleatoria de unidades primarias (transectos) y el establecimiento arbitrario de unidades secundarias (parcelas). Las parcelas se distribuyeron contiguamente, siempre desde el área del borde hacia el interior del fragmento, contemplando un gradiente ambiental (Krahl et al., 2010; Souza-Leal \& Pedroso-de-Moraes, 2014). El criterio para la inclusión de individuos en la parcela fue el enraizamiento de la planta en el sitio (Souza et al., 2012), por lo que los individuos que se extienden a más de una parcela se contaron solo una vez. Como $V$. bahiana germina y llega a la edad adulta sin depender de un árbol huésped, no se evaluaron los forófitos sobre los que se apoyaba. Tampoco se verificó la diferenciación entre individuos juveniles y adultos (individuos en edad reproductiva) de $V$. bahiana en APA Lagoas y Dunas do Abaeté, ya que solo los individuos (o partes de ellos) ubicados en áreas de mayor luminosidad, como los bordes de los restos de vegetación, tienden a florecer (Anjos et al., 2017), lo que podría no reflejar la etapa de maduración de los individuos.
Para identificar el patrón de distribución espacial, se aplicó el índice de Morisita (Im), que es considerado uno de los mejores índices de dispersión y adecuados para el análisis con transectos, y también el índice de Morisita estandarizado (Ip), ya que es relativamente poco influenciado por el número de muestras y tamaño de las unidades de muestreo (Krebs, 2014; Oksanen et al., 2019).

Para el Im, los valores estadísticamente significativos $>1$ indican agregación, valores $<1$ reflejan uniformidad y $\mathrm{Im}=1$ mostra un patrón aleatorio. La significación estadística se infirió mediante la prueba de $\chi^{2}$ (Chi cuadrado), con un nivel de significación de 0,05 y los correspondientes grados de libertad $(n-1)$. Para obtener el Ip, se calculó previamente el Im y sus dos valores críticos, el índice de uniformidad $(\mathrm{Mu})$ y el índice de agregación (Mc). Para Ip, los valores entre $-0,5$ y 0,5 corresponden a una distribución aleatoria de la población, valores menores o iguales que $-0,5$ indican una distribución uniforme y valores mayores 
o iguales que 0,5 expresan agregación (con 95\% de límites de confianza) (Oksanen et al., 2019).

De forma complementaria y para evitar estimaciones sesgadas, derivadas del uso de índice similares y basados en la independencia de las muestras, también se utilizó el método de varianza móvil entre bloques de parcelas (TTLQV), recomendado para detectar la intensidad del patrón espacial (oscilación de densidad en la muestra) (Dale, 1999). La determinación del patrón de distribución se realizó a través de la interpretación del gráfico generado a partir del cálculo de las varianzas de conjuntos agrupados de cuadrantes adyacentes. Si las varianzas fluctúan, mostrando un pico en un tamaño de bloque (equivalente al radio del tamaño del grupo), el patrón es agregado; si las varianzas estimadas son todas bajas y no fluctúan con el tamaño del bloque, el patrón es uniforme; y si los valores de varianza fluctúan irregularmente, el patrón es aleatorio (Krebs, 2014).

Aunque TTLQV asume la contigüidad de las unidades de muestreo, se realizó un primer análisis relativizando esta premisa, y considerando los transectos de cada fitofisionomía (y sus respectivas parcelas) como contiguos (en la secuencia de transectos 1-6 para cada formación). Se adoptó el límite superior recomendado del tamaño del bloque de ${ }^{n / 10}$ (Krebs, 2014). Debido a que los transectos no son realmente muestras independientes de la población, se realizó también un análisis de TTLQV para cada transecto. Sin embargo, adoptamos $n / 2$ como el límite superior del tamaño del bloque debido al número reducido de unidades de muestreo.

\section{RESULTADOS}

La distribución espacial de $V$. bahiana fue evaluada en dos fitofisionomías de restinga. Se contaron 49 individuos de $V$. bahiana en la formación de arbustos $(8,17 \pm 8,49$ individuos por transecto) y 47 individuos en el bosque de restinga $(7,83 \pm 3,31$ individuos por transecto). En la fitofisiognomía de arbusto hubo una mayor variación en la densidad de $V$. bahiana entre los transectos, que fluctuó de 0,01875 individuos $/ \mathrm{m}^{2}$ a 0,13125 individuos $/ \mathrm{m}^{2}$ (3 a 21 individuos por transecto), mientras que en la formación de bosque la variación fue menor, de 0,0125 individuos $/ \mathrm{m}^{2}$ a 0,075 individuos $/ \mathrm{m}^{2}$ ( 2 a 12 individuos por transecto). De las 60 parcelas establecidas en la AR, 14 presentaron individuos de $V$. bahiana $(23,3 \%$ de frecuencia absoluta), con una clara concentración de individuos en el borde de los transectos (parcelas 1 y 2), correspondientes a aproximadamente $82 \%$ del total de especímenes reportados para la fitofisionomía. En contraste, no se observaron individuos en las parcelas 8 a 10 en ninguno de los transectos de AR (Fig. 3A). En la formación de BR, los individuos se encontraron en 34 parcelas (frecuencia absoluta del 56,6\%), con una distribución más homogénea, habitando tanto parcelas en los bordes como en el interior de los transectos (Fig. 3B).

La varianza y la abundancia observadas resultaron en un patrón de distribución agregada para $V$. bahiana en la formación de AR y un patrón aleatorio en BR. Para la formación de AR, se encontró IM = 1,82 , con un estadístico de contraste $\chi^{2}=44,18$, muy
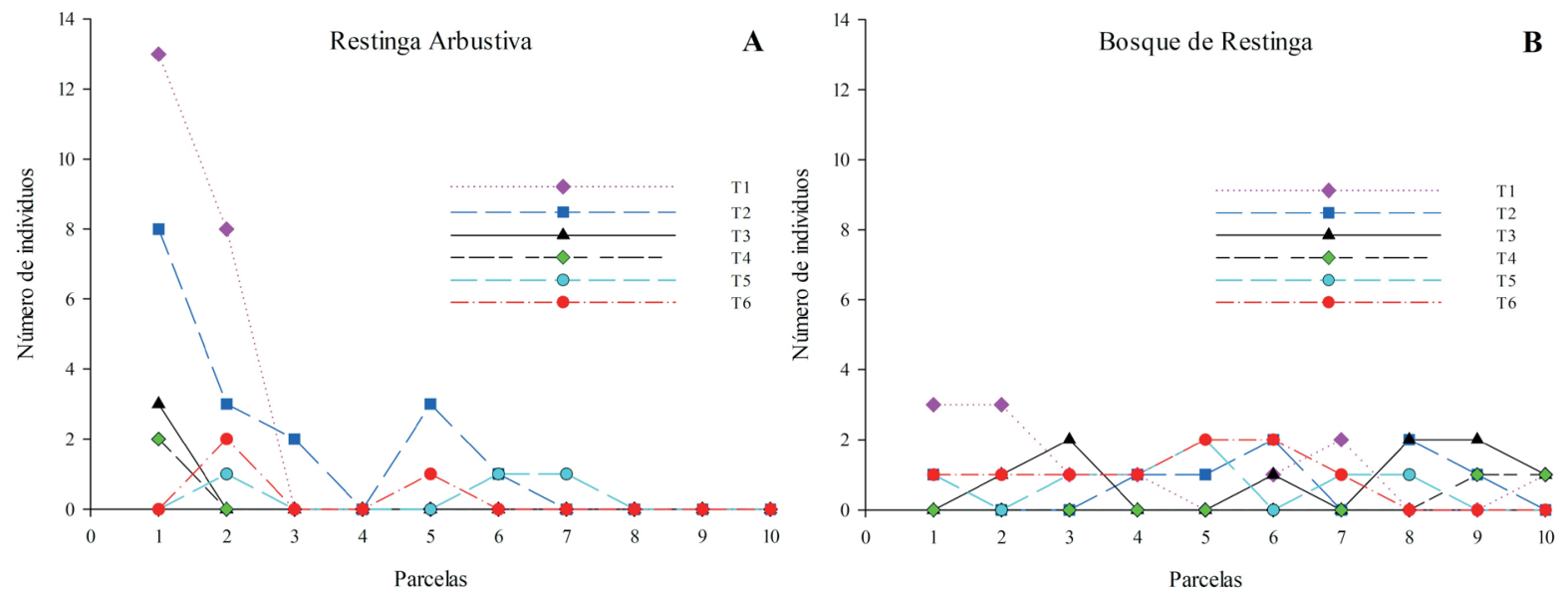

Figura 3. Abundancia de Vanilla bahiana por parcelas en el Parque Municipal Abaeté y sus alrededores, Bahía, Brasil: (A), en la formación arbustiva; (B), en el bosque de restinga. 
por encima del valor tabulado de 11,07. También se obtuvieron $\mathrm{Mu}=0,91, \mathrm{Mc}=1,16$ e Ip $=0,57$. El valor de Im fue superior a 1 y el valor de Ip fue superior a 0,5 , resultados que indican distribución agregada. En la formación de BR, se obtuvo $\mathrm{IM}=1,04$. Sin embargo, el estadístico de contraste no difiere de lo esperado al azar, y, por lo tanto, la distribución de los individuos de $V$. bahiana en BR corresponde a un patrón aleatorio. Esta inferencia fue confirmada a través del Ip. Para la formación de BR, se encontraron $\mathrm{Mu}=0,91, \mathrm{Mc}=1,17 \mathrm{e} \mathrm{Ip}=0,13$.

Los resultados de TTLQV para transectos contiguos en la AR permitieron resaltar la gran oscilación en la varianza (valores de 2 a 3,9 ) en relación con los tamaños de bloque adoptados, con todos los valores por encima del promedio de 0,82 , y un pico muy evidente en el tamaño de bloque 2 y otro pico menos patente en el tamaño de bloque 5, que son indicativos de agregación (Fig. 4). Para la formación de BR se obtuvieron valores de varianza menos discrepantes, de 0,45 a 0,78 , e iguales o cercanos al promedio $(0,78)$, sin la formación de picos, correspondiendo a una distribución aleatoria (Fig. 4).

A su vez, el análisis de los resultados de TTLQV por transecto permitió detectar patrones distintos dentro de la formación de arbustos. Hubo una agregación clara en los transectos 1 y 2 , representada por una alta varianza (muy por encima del promedio para todos los tamaños de bloque) y picos muy claros, especialmente para los tamaños de bloque 4 y 5 . Sin embargo, en los transectos 3 a 6 no se observaron picos y los valores de varianza en relación

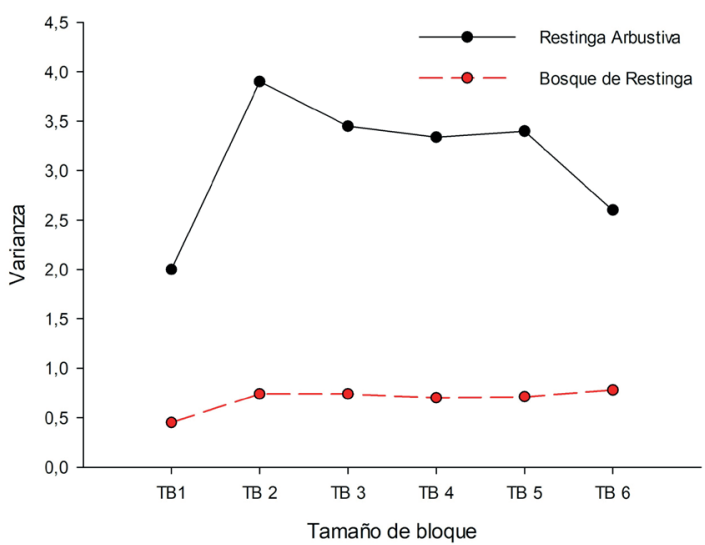

Figura 4. Análisis de la estructura espacial de Vanilla bahiana en el Parque Municipal Abaeté y alrededores, Bahía, Brasil, según el método TTLQV y considerando los transectos de cada fitofisionomía como contiguos: línea negra contínua, en la formación arbustiva; línea roja discontínua, en el bosque de restinga.

con los tamaños de los bloques variaron poco, no excediendo de 0,9 , y permanecieron cerca de los respectivos promedios de los transectos (Fig. 5A). Así, es claro que el patrón agregado observado en la formación arbustiva es el resultado de la mayor abundancia y distribución de individuos en los transectos 1 y 2. Para la formación del bosque de restinga, sin embargo, se observaron patrones similares para todos los transectos, marcados por la fluctuación poco acentuada e irregular de los valores de varianza, característica de la distribución aleatoria (Fig. 5B).
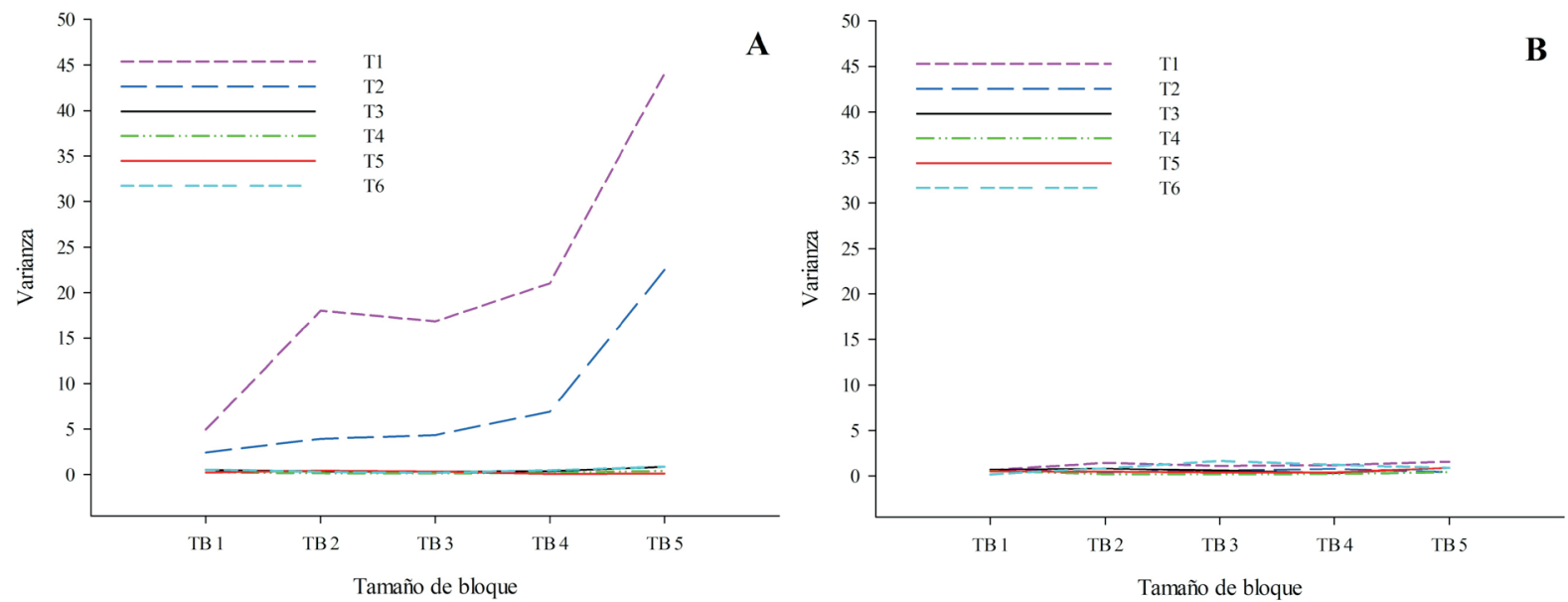

Figura 5. Análisis de la estructura espacial de Vanilla bahiana en el Parque Municipal Abaeté y alrededores, Bahía, Brasil, según el método TTLQV y considerando los transectos de cada fitofisionomía como unidades muestrales no contiguas: (A), en la formación arbustiva; (B), en el bosque de restinga. 


\section{DISCUSIÓN}

Se encontró que el patrón de distribución espacial de una especie de orquídea puede variar en diferentes fitofisiognomías en la misma área. En la formación de arbustos, verificamos el patrón agregado para $V$. bahiana, pero en el bosque de restinga encontramos el patrón aleatorio. Dichos patrones fueron los mismos para los tres índices empleados. Lima-Ribeiro \& Prado (2006) demostraron para especies de Annonaceae y Asteraceae que la adopción de diferentes métodos (sea los que utilizan el número de individuos por parcela o aquellos que estiman la varianza entre diferentes combinaciones de parcelas) para el análisis del grado de agrupamiento de una población no resulta en patrones espaciales distintos. Por lo tanto, los resultados encontrados en el presente estudio pueden considerarse consistentes. Además, varios autores han observado fluctuaciones (aumento o disminución) en los valores de Im al cambiar el tamaño de las parcelas de ocurrencia de orquídeas terrestres, tanto para individuos inmaduros (que no muestran evidencia de actividad reproductiva durante el período de observación) como maduros (que muestran evidencia reproductiva), pero siempre sin alteración del patrón observado (Budke et al., 2004; Piccoli et al., 2014; Souza-Leal \& Pedroso-de-Moraes, 2014; Pedroso-de-Moraes \& Souza-Leal, 2015).

El patrón agregado encontrado para $V$. bahiana en la formación AR es repetitivo en Orchidaceae en el Neotrópico y se observa tanto en individuos inmaduros como en maduros de la misma especie y también en áreas de muestreo de diferentes tamaños (p. ej. Brudke et al., 2004; Otero et al., 2007a; Carvalho et al., 2012; Souza-Leal \& Pedroso-de-Moraes, 2014; Rahal et al., 2015). El patrón agregado se encontró para varias orquídeas terrestres en los tipos de vegetación del bosque atlántico, incluidas las formaciones de arbustos y bosques de restinga (Krahl et al., 2010; Souza et al., 2012). Adicionalmente, Carvalho et al. (2012) también reconocieron el patrón agregado para $V$. bahiana en un fragmento de Caatinga en el estado de Bahía, aunque el número de individuos observados fue bajo $(n=10)$.

La agrupación es favorecida por la presencia de uno o más recursos favorables para el desarrollo de los individuos y la restinga es un tipo de vegetación heterogéneo con parches apropiados (vegetación) y no apropiados (arena desnuda) para la ocurrencia de la especie. Del mismo modo, principalmente en la formación arbustiva, observamos parcelas con agrupación de individuos de $V$. bahiana (ocho o trece) y otras con pocos individuos (uno o dos) o incluso deshabitadas, ya que pueden constituir manchas desfavorables, proporcionando un equilibrio entre la colonización de hábitats disponibles y extinción de subpoblaciones locales (Tremblay et al., 2006).

Uno de los requisitos para la germinación en $\mathrm{Or}$ chidaceae está estrictamente relacionado con la formación de asociaciones simbióticas con hongos micorrícicos (predominantemente especies de Basidiomycota) desde el comienzo de su ciclo de vida (Feuerherdt et al., 2005; Dearnaley, 2007). PorrasAlfaro \& Bayman (2007) demostraron que el género Vanilla es generalista para hongos micorrícicos y que los hongos asociados con la germinación en Vanilla son especies diferentes de las que ocurren en otras etapas del ciclo de vida de las orquídeas de este género. Estos autores también observaron que la presencia de hongos micorrícicos en las raíces de especies de vainilla en el suelo es mayor que en las raíces en la corteza de los árboles y que el género Xenasma Donk (Ceratobasidium D. P. Rogers) es el más común en raíces en el suelo y el único en promocionar la germinacion de semillas de vainilla in vitro. En el suelo y en los forófitos, los hongos micorrícicos también tienen una distribución agregada $\mathrm{y}$, aunque tienen una distribución independiente de la presencia de orquídeas (Feuerherdt et al., 2005; McCormick \& Jacquemyn, 2013), se encuentran poblaciones más grandes de hongos cerca de tales plantas (Perkins \& McGee, 1995; Batty et al., 2001; Diez, 2007). Como el suelo es heterogéneo en ambientes forestales se supone que la disponibilidad de sustrato para hongos también varía a microescala (Diez, 2007). Por lo tanto, la disponibilidad de hongos compatibles es un factor determinante para la presencia, abundancia y distribución de orquídeas en un determinado lugar. Este aspecto podría explicar la variación entre los patrones de distribución de $V$. bahiana en las dos formaciones de vegetación.

Las especies de Vanilla poseen semillas recalcitrantes y crustosas con endospermo, y posible dispersión zoocórica; abejas, murciélagos frugívoros y aves son sugeridos como agentes dispersantes de semillas en el género, además del viento asociado a la gravedad (Cameron \& Chase, 1998; Householder et 
al., 2010; Soto-Arenas \& Dressler, 2010). Aunque se produce la maduración del fruto de $V$. bahiana en la APA Abaeté (Anjos et al., 2017; Nascimento et al., 2019), no se observan plántulas y la forma de dispersión de semillas en $V$. bahiana sigue siendo desconocida. Contrariamente a la percepción común, la dispersión de semillas de orquídeas por el viento ocurre en distancias cortas de menos de 10 metros (Murren \& Ellison, 1998; Chung et al., 2004). Además, la dispersión de semillas por el viento a partir de áreas abiertas hacia áreas de vegetación más cerradas puede ser difícil (Azevedo et al., 2007), como a partir de la formación arbustiva, y puede contribuir a generar el patrón aleatorio observado en los bosques de restinga. Igualmente, la dispersión de semillas por animales en la formación de BR puede ser ineficiente para la formación de nuevos rodales de individuos, ya que la circulación de dispersores en sitios más cerrados es más difícil.

Además de la dispersión de semillas y de las relaciones micorrícicas, otro factor que probablemente interfiere con la distribución de $V$. bahiana en la restinga es la luminosidad. Los hongos micorrícicos son más eficientes en la colonización cuando son expuestos a alta intensidad de luz (igual o mayor a $600 \mu \mathrm{mol} \mathrm{m} \mathrm{m}^{-2} \mathrm{~s}^{-1}$; Moratelli et al., 2007), lo que, a su vez, podría explicar las distintas microdistribuciónes de $V$. bahiana en las dos fitofisiognomías. En la formación AR, se encontró un mayor número de individuos en los bordes de los transectos (parcelas 1 y 2), una región de luminosidad más intensa, con menor cobertura vegetal, mientras que, en BR, no se observó una mayor abundancia en las parcelas fronterizas o interiores (Fig. 3). El dosel heterogéneo de AR proporcionaría una mayor luminosidad en algunas parcelas, posiblemente cerca del óptimo para la eficiencia de la colonización por hongos. Aunque no se obtuvieron los datos abióticos para cada parcela, la mayor presencia de individuos en las parcelas de borde asume que la luz (y posiblemente la humedad) pueden explicar la distribución en puntos de $V$. bahiana en la fitofisionomía de arbustos. En contraste, el dosel homogéneo de BR resultaría en una menor penetración de luz y diferencias de luz menos significativas entre las parcelas, haciendo que los microclimas en esta fitofisionomía sean similares.

El patrón de distribución espacial en Orchidaceae cambia según las etapas ontogenéticas, pasando de agregado a aleatorio o uniforme, bajo una mayor influencia de las interacciones bióticas, como la competencia inter e intraespecífica, herbívoros y patógenos, y con el reclutamiento a una distancia más espaciada de la planta madre (Souza et al., 2012). Por lo tanto, los diferentes patrones encontrados para $V$. bahiana podrían explicarse por diferencias de luminosidad en las fitofisonomías, estar relacionados con agentes dispersantes de semillas o con la presencia de individuos de orquídeas en diferentes etapas del ciclo de vida, influenciados por la presencia de hongos de diferentes especies. Así, los individuos de $V$. bahiana de las formaciones forestales pueden estar en una etapa más avanzada de desarrollo que los individuos de las formaciones de arbustos, presentando un patrón aleatorio, resultante de la mayor influencia de las interaciones bióticas y reflejando una homogeneidad ambiental.

Independientemente de los factores responsables de estos patrones, nuestros hallazgos permitieron definir áreas de mayor y menor densidad poblacional y detectar discontinuidades en la distribución a pequeña escala de $V$. bahiana, lo que nos permitirá adoptar estrategias de refuerzo de la población más detalladas para la especie en la APA Abaeté y posiblemente extrapolarlas a áreas de restinga adyacentes.

\section{CONSIDERACIONES FINALES}

Varios autores indican que el patrón de distribución espacial en Orchidaceae se relaciona primariamente con la distribución y abundancia de hongos micorrícicos, aunque la distribución de orquídeas también pueda ser determinada por factores adicionales (Feuerherdt et al., 2005; Otero et al., 2007b; Waterman \& Bidartondo, 2008; McCormick \& Jacquemyn, 2013). De manera similar a lo verificado para las orquídeas terrestres, la especificidad de las orquídeas epífitas por ciertos forófitos puede ser un reflejo de la asociación entre hongos y especies arbóreas (Otero et al., 2007a; Gowland et al., 2011) y, por lo tanto, la distribución del hongo sería el factor limitante para la distribución de las orquídeas epífitas y no el forófito en sí. A su vez, la distribución y abundancia de hongos micorrícicos también puede depender de factores bióticos y abióticos, incluyendo variaciones en microescala en las condiciones edáficas o de sustrato, lo que hace que la comprensión de las causas de los patrones espaciales de las 
orquídeas sea aún más compleja (Osorio-Gil et al., 2008; McCormick \& Jacquemyn, 2013). Ciertamente hay mucho que conocer sobre la actividad de forrajeo del hongo en habitats naturales y sobre métodos de almacenamiento y cultivo de hongos micorrícicos, así como sobre los transportadores de nutrientes y genes relacionados con el mantenimiento de la asociación simbiótica (Batty et al., 2001; Dearnaley, 2007).

Bajo este sesgo, los estudios futuros con distribución espacial en Orchidaceae en microescalas deberían ser pluridisciplinares, centrados en la investigación de las causas de los patrones y desarollados en línea con los estudios de la distribución de los hongos micorrícicos, ya que estos podrían tener influencia directa en la comprensión de la biología, ecología, estrutura genética y acciones de conservación o restauración que involucren las orquídeas, siendo especialmente importante para especies raras, amenazadas de extinción o endémicas como $V$. bahiana. Específicamente, comprender las relaciones entre especies de Vanilla y los hongos micorrícicos puede generar impactos positivos sobre el cultivo y la comercialización de vainilla.

\section{AGRADECIMIENTOS}

Agradecemos al Sr. J. Santana, gerente del Parque Municipal das Dunas, por la autorización otorgada para realizar este estudio, y a J. A. Lima Rocha Junior, por la elaboración del mapa y de los gráficos.

\section{REFERENCIAS}

Alvares, C. A., Stape, J. L., Sentelhas, P. C., Gonçalves, J. L. M. \& Sparovek, G. 2013. Köppen's climate classification map for Brazil. Meteorologische Zeitschrift 22: 711-728. https://doi.org/10.1127/0941-2948/2013/0507

Anjos, A., Mazza, M. C. M., Santos, A. C. M. C. \& Delfini, L. T. 2004. Análise do padrão de distribuição espacial da araucária (Araucaria angustifolia) em algumas áreas no estado do Paraná, utilizando a função K de Ripley. Scientia Forestalis 66: 38-45.

Anjos, A. M., Barberena, F. F. V. A. \& Pigozzo, C. M. 2017. Biologia reprodutiva de Vanilla bahiana Hoehne (Orchidaceae). Orquidário 30: 67-79.

Azevedo, M. T. A., Borba, E. L., Semir, J. \& Solferini, V. N. 2007. High genetic variability in Neotropical myophilous orchids. Botanical Journal of the Linnean Society 153: 33-40. https://doi.org/10.1111/j.1095-8339.2007.00589.x

Barberena, F. F. V. A., Baumgratz, J. F. A. \& Barros, F. 2018. Ecological data for an orchid diversity hotspot show that the subtribe Laeliinae may be endangered in the Brazilian
Atlantic Forest. Nordic Journal of Botany 36: e01728. https://doi.org/10.1111/njb.01728

Barberena, F. F. V. A., Sousa, T. S. \& Rocha Junior, J. A. L. 2019. Mapping threats to the orchid populations in an environmental protection area in Bahia, Northeast Brazil. Oecologia Australis 23: 346-356. https://doi.org/10.4257/ oeco.2019.2302.12

Barros, F. 2007. A família Orchidaceae na Flora do Estado de São Paulo e suas espécies ameaçadas. In: Mamede, M. C. H., Souza, V. C., Prado, J., Wanderley, M. G. L., Barros, F. \& Rando, J. G. (Eds.), Livro vermelho das espécies vegetais ameaçadas do estado de São Paulo. Instituto de Botânica, São Paulo: 47-52.

Batty, A. L., Dixon, K. W. \& Sivasithamparam, K. 2001. Constraints to symbiotic germination of terrestrial orchid seed in a Mediterranean bushland. New Phytologist 152: 511520. https://doi.org/10.1046/j.0028-646X.2001.00277.x

Bonet, A., Gras, M. J. \& Raventós, J. 2005. Análisis de los patrones espaciales de distribución de Vella lucentina M. B. Crespo para la selección de áreas de la Red de Microrreservas de Flora de la Comunidad Valenciana. Mediterránea: Serie de Estudios Biológicos 18: 8-43.

Bouetard, A., Lefeuvre, P., Gigant, R., Bory, S., Pignal, M., Besse, P. \& Grisoni, M. 2010. Evidence of transoceanic dispersion of the genus Vanilla based on plastid DNA. Molecular Phylogenetics and Evolution 55: 621-630. https:// doi.org/10.1016/j.ympev.2010.01.021

Britto, I. C., Queiroz, L. P., Guedes, M. L. S., Oliveira, N. C. \& Silva, L. B. 1993. Flora Fanerogâmica das Dunas e Lagoas do Abaeté, Salvador, Bahia. Sitientibus 11: 31-46.

Budke, J. C., Giehll, L. H., Athaydel, E. A. \& Záchial, R. A. 2004. Distribuição espacial de Mesadenella cuspidata (Lindl.) Garay (Orchidaceae) em uma floresta ribeirinha em Santa Maria, RS, Brasil. Acta Botanica Brasilica 18: 3135. https://doi.org/10.1590/S0102-33062004000100004

Bythrow, J. D. 2005. Vanilla as a medicinal plant. Seminars in Integrative Medicine 3: 129-131. https://doi.org/10.1016/j. sigm.2006.03.001

Cameron, K. M. \& Chase, M. W. 1998. Seed morphology of vanilloid orchids (Vanilloideae: Orchidaceae). Lindleyana 13: $148-169$.

Carvalho, D. N., Berg, C. van den \& Pigozzo, C. M. 2012. Floristic and spatial distribution of Orchidaceae species in the Serra do Mucambo, Conceição do Coité, Bahia, Brazil. Brazilian Journal of Ecology 14: 66-73.

Chung, M. Y. \& Chung, M. G. 1999. Allozyme diversity and population structure in Korean populations of Cymbidium goeringii (Orchidaceae). Journal of Plant Research 112: 139-144. https://doi.org/10.1007/PL00013868

Chung, M. Y., Nason, J. D. \& Chung, M. G. 2004. Spatial genetic structure in populations of the terrestrial orchid Cephalanthera longibracteata (Orchidaceae). American Journal of Botany 91: 52-57. https://doi.org/10.3732/ajb.91.1.52

Dale, M. R. T. 1999. Spatial pattern analysis in plant ecology. Cambridge University Press, Cambridge. https://doi. org/10.1017/CBO9780511612589

Dearnaley, J. D. W. 2007. Further advances in orchid mycorrhizal research. Mycorrhiza 17: 475-486. https://doi. org/10.1007/s00572-007-0138-1

Diário Oficial do Estado da Bahia 1987. Decreto $n^{\circ} 351$, de 22 de setembro de 1987. Retrieved April 29, 2020, from http://www.icmbio.gov.br/cepsul/images/stories/legislacao/Decretos/1987/dec_ba_351_1987_uc_criaapa_lagoas dunasabaete_salvador_ba_altrd_dec_2540_1993_res_cepram_3023_2002.pdf/ 
Diário Oficial do Município de Salvador 2011. Decreto $n^{\circ}$ 22.507, de 22 de dezembro de 2011. Retrieved April 7, 2020, from https://leismunicipais.com.br/a/ba/s/salvador/ decreto/2011/2250/22507/decreton-22507-2011-delimita-e-implanta-o-parquedas-dunas-amplia-o-parque-do-abaete-e-daoutras-providencias

Diez, J. M. 2007. Hierarchical patterns of symbiotic orchid germination linked to adult proximity and environmental gradients. Journal of Ecology 95: 159-170. https://doi. org/10.1111/j.1365-2745.2006.01194.x

Dubbern, C. A., Leal, T. S. \& Pedroso-de-Moraes, C. 2013. Distribuição espacial de Oeceoclades maculata Lindl. (Orchidaceae) em fragmento florestal estacional semidecidual da fazenda Santa Tereza, Cordeirópolis, SP, Brasil. Natureza on line 11: 29-32.

Fajardo, C. G., Costa, R. A., Vieira, F. A. \& Molina, W. F. 2015. Distribuição espacial de Cattleya granulosa: uma orquídea ameaçada de extinção. Floresta e Ambiente 22: 164-170. https://doi.org/10.1590/2179-8087.073714

Ferreira, A. W. C., Oliveira, M. S., Silva, E. O., Campos, D. S., Pansarin, E. R. \& Guarçoni, E. A. E. 2017. Vanilla bahiana Hoehne and Vanilla pompona Schiede (Orchidaceae, Vanilloideae): two new records from Maranhão state, Brazil. Check List 13: 1131-1137. https://doi. org/10.15560/13.6.1131

Feuerherdt, L., Petit, S. \& Jusaitis, M. 2005. Distribution of mycorrhizal fungus associated with the endangered pinklipped spider orchid (Arachnorchis (syn. Caladenia) behrii) at Warren Conservation Park in South Australia. New Zealand Journal of Botany 43: 367-371. https://doi.org/10.108 0/0028825X.2005.9512961

Flora do Brasil 2020. Vanilla bahiana. Jardim Botânico do Rio de Janeiro, Rio de Janeiro. Retrieved April 29, 2020, from http://floradobrasil.jbrj.gov.br/reflora/floradobrasil/FB12349

Gowland, K. M., Wood, J., Clements, M. A. \& Nicotra, A. B. 2011. Significant phorophyte (substrate) bias is not explained by fitness benefits in three epiphytic orchid species. American Journal of Botany 98: 197-206. https://doi. org/10.3732/ajb.1000241

Hay, J. D., Bizerril, M. X., Calouro, A. M., Costa, E. M. N., Ferreira, A. A., Gastal, M. L. A., Goes Junior, C. D., Manzan, D. J., Martins, C. R., Monteiro, J. M. G., Oliveira, S. A., Rodrigues, M. C. M., Seyffarth, J. A. S. \& Walter, B. M. T. 2000. Comparação do padrão da distribuição espacial em escalas diferentes de espécies nativas do cerrado, em Brasília, DF. Revista Brasileira de Botânica 23: 341-347. https://doi.org/10.1590/S0100-84042000000300008

Householder, E., Janovec, J., Mozambite, A. B., Maceda, J. H., Wells, J. \& Valega, R. 2010. Diversity, natural history, and conservation of Vanilla (Orchidaceae) in Amazonian wetlands of Madre de Dios, Peru. Botanical Research Institute of Texas 4: 227-243.

Hutchings, M. J. 1997. The structure of plant populations. In: Crawley, M. J. (Ed.), Plant ecology. Blackwell Scientific Publications, Oxford: 325-358. https://doi. org/10.1002/9781444313642.ch11

Jacquemyn, H., Brys, R., Vandepitte, K., Honnay, O., RoldánRuiz, I. \& Wiegand, T. 2007. A spatially explicit analysis of seedling recruitment in the terrestrial orchid Orchis purpurea. New Phytologist 176: 448-459. https://doi. org/10.1111/j.1469-8137.2007.02179.x

Kanieski, M. R., Longhi, S. L., Narvaes, I. S., Soares, P. R. C., Longhi-Santos, T. \& Callegaro, R. M. 2012. Diversidade e padrões de distribuição espacial de espécies no estágio de regeneração natural em São Francisco de Paula, RS, Brasil. Floresta 42: 509-518. https://doi.org/10.5380/rf. v42i3.25037

Krahl, A. H., Souza, G. R., Boldrini, R. F., Carneiro, B. M., Duca, C. \& Azevedo-Júnior, R. R. 2010. Padrão espacial de Eltroplectris triloba (Lindl.) Pabst (Orchidaceae, Spiranthinae) em uma formação florestal de restinga no sudeste do Brasil. Orquidário 24: 117-123.

Krebs, C. J. 2014. Ecological methodology. Benjamin Cummings, San Francisco.

Lima-Ribeiro, M. S. \& Prado, E. C. 2006. Métodos estatísticos e estrutura espacial de populações: uma análise comparativa. Acta Scientiarum. Technology 28: 219-224. https://doi. org/10.4025/actascitechnol.v28i2.1197

Lopes, E. M., Linhares, R. G., Pires, L. O., Castro, R. N., Souza, G. H. M. F., Koblitz, M. G. B., Cameron, L. C. \& Macedo, A. F. 2019. Vanilla bahiana, a contribution from the Atlantic Forest biodiversity for the production of vanilla: A proteomic approach through high-definition nanoLC/MS. Food Research International 120: 148-156. https://doi. org/10.1016/j.foodres.2019.02.038

McCormick, M. K. \& Jacquemyn, H. 2013. What constrains the distribution of orchid populations? New Phytologist 202: 392-400. https://doi.org/10.1111/nph.12639

Moratelli, E. M., Costa, M. D., Lovato, P. E., Santos, M. \& Paulilo, M. T. S. 2007. Efeito da disponibilidade de água e de luz na colonização micorrízica e no crescimento de Tabebuia avellanedae Lorentz ex Griseb. (Bignoniaceae). Revista Árvore 31: 555-566. https://doi.org/10.1590/ S0100-67622007000300021

MMA [Ministério do Meio Ambiente do Brasil] 2012. Resolução Conama $n^{\circ} 437$, de 30 de dezembro de 2011. Retrieved April 30, 2020, from http://www.mma.gov.br/port/conama/ res/res12/Resol437.pdf

Murren, C. J. \& Ellison, A. M. 1998. Seed dispersal characteristics of Brassavola nodosa (Orchidaceae). American Journal of Botany 85: 675-680. https://doi.org/10.2307/2446537

Nascimento, T. A., Furtado, M. S. C., Pereira, W. C. \& Barberena, F. F. V. A. 2019. Vanilla bahiana Hoehne (Orchidaceae): studies on fruit development and new perspectives into crop improvement for the Vanilla planifolia group. Biota Neotropica 19: e20180696. https://doi.org/10.1590/16760611-bn-2018-0696

Oksanen, J., Blanchet, F. G., Friendly, M., Kindt, R., Legendre, P., McGlinn, D., Minchin, P. R., O’Hara, R. B., Simpson, G. L., Solymos, P., Stevens, M. H. H., Szoecs, E. \& Wagner, H. 2019. Vegan: Community Ecology Package. R package version 2.5-6. Retrieved November 3, 2020, from https:// cran.r-project.org/web/packages/vegan/index.html

Osorio-Gil, E. M., Forero-Montaña, J. \& Otero, J. T. 2008. Variation in mycorrhizal infection of the epiphytic orchid Ionopsis utriculariodes (Orchidiaceae) on different substrata. Caribbean Journal of Science 44: 130-132. https://doi. org/10.18475/cjos.v44i1.a15

Otero, J. T., Aragón, S. \& Ackerman, J. D. 2007a. Site variation in spatial aggregation and phorophyte preference in Psychilis monensis (Orchidaceae). Biotropica 39: 227-231. https://doi.org/10.1111/j.1744-7429.2006.00258.x

Otero, J. T., Flanagan, N. S., Herre, E. A., Ackerman, J. D. \& Bayman, P. 2007b. Widespread mycorrhizal specificity correlates to mycorrhizal function in the Neotropical, epiphytic orchid Ionopsis utricularioides (Orchidaceae). American Journal of Botany 94: 1944-1950. https://doi.org/10.3732/ ajb.94.12.1944 
Pandey, M., Sharma, J., Donald, L. T. \& Yadon, V. L. 2013. A narrowly endemic photosynthetic orchid is non-specific in its mycorrhizal associations. Molecular Ecology 22: 2341-2354. https://doi.org/10.1111/mec.12249

Pedroso-de-Moraes, C. \& Souza-Leal, T. 2015. Habenaria repens: distribuição espacial e correlação com fatores abióticos de uma orquídea emergente invasora em fragmento mesófilo brasileiro. Revista em Agronegócio e Meio Ambiente 8: 111-136. https:// doi.org/10.17765/2176-9168.2015v8nEd.esp.p111-136

Peixoto, A. L. \& Maia, L. C. 2013. Manual de procedimentos para herbários. Editora Universitária UFPE, Recife.

Perkins, A. J. \& McGee, P. A. 1995. Distribution of the orchid mycorrhizal fungus, Rhizoctonia solani, in relation to its host, Pterostylis acuminata, in the field. Australian Journal of Botany 43: 565-575. https://doi.org/10.1071/BT9950565

Phillips, R. D., Brown, A. P., Dixon, K. W. \& Hopper, S. D. 2010. Orchid biogeography and factors associated with rarity in a biodiversity hotspot, the Southwest Australian Floristic Region. Journal of Biogeography 38: 487-501. https://doi.org/10.1111/j.1365-2699.2010.02413.x

Piccoli, M. C. A., Souza-Leal, T. de \& Pedroso-de-Moraes, C. 2014. Distribuição espacial de Sacoila lanceolata (Aubl.) Garay (Orchidaceae) em fragmento mesófilo de Pirassununga, São Paulo, Brasil. Nucleus 11: 115-130. https://doi. org/10.3738/1982.2278.937

Porras-Alfaro, A. \& Bayman, P. 2007. Mycorrhizal fungi of Vanilla: diversity, specificity and effects on seed germination and plant growth. Mycologia 99: 510-525. https://doi. org/10.3852/mycologia.99.4.510

Quenzer, F. C. L. \& Pedroso-de-Moraes, C. 2014. Distribuição espacial de Oeceoclades maculata Lindl. em fragmento florestal estacional semidecidual do "Sítio João XXIII", Pirassununga, SP, Brasil. Natureza on line 12: 160-163.

Rahal, M. H., Souza-Leal, T. \& Pedroso-de-Moraes, C. 2015. Ecologia e distribuição espacial de orquídeas terrícolas em floresta estacional semidecidual do município de Araras, São Paulo, Brasil. Iheringia 70: 217-228.

Soto-Arenas, M. A. \& Dressler, R. L. 2010. A revision of the Mexican and Central American species of Vanilla Plumier ex Miller with a characterization of their ITS region of the nuclear ribosomal DNA. Lankesteriana 9: 285-354.

Sousa, D. G. \& Cunha, H. F. 2018. Population structure, spatial distribution and phenology of Anacardium humile A. St.Hil. (Anacardiaceae) in cerrado stricto sensu. Hoehnea 45: 450-467. https://doi.org/10.1590/2236-8906-81/2017

Souza, G. R., Krahl, A. H. \& Silva, A. G. 2012. Population structure and spatial pattern of Catasetum discolor (Lindl.) Lindl. (Orchidaceae) in a sandy coastal plain southeastern Brazil. Natureza on line 10: 105-110.

Souza-Leal, T. \& Pedroso-de-Moraes, C. 2014. Fenologia reprodutiva e distribuição espacial de Oeceoclades maculata (Lindl.) Lindl. (Orchidaceae) em Cerrado do município de Mogi Guaçu, São Paulo, Brasil. Iheringia 69: 405-416.

Teixeira, N. D. A., Marimon, B. S., Elias, F. \& Marimon-Junior, B. H. 2019. Padrões espaciais de samambaias em floresta estacional perenifólia na transição Amazônia-Cerrado. Rodriguésia 70: e02572016. https://doi.org/10.1590/21757860201970011

Tremblay, R. L., Meléndez-Ackerman, E. \& Kapan, D. 2006. Do epiphytic orchids behave as metapopulations? Evidence from colonization, extinction rates and asynchronous population dynamics. Biological Conservation 129: 70-81. https://doi.org/10.1016/j.biocon.2005.11.017

Viana, B. F. \& Kleinert, A. M. P. 2005. A community of flower-visiting bees (Hymenoptera: Apoidea) in the coastal sand dunes of Northeastern Brazil. Biota Neotropica 5: 1-13. https://doi.org/10.1590/S1676-06032005000300005

Waterman, R. J. \& Bidartondo, M. I. 2008. Deception above, deception below: linking pollination and mycorrhizal biology of orchids. Journal of Experimental Botany 59: 10851096. https://doi.org/10.1093/jxb/erm366

Watteyn, C., Fremout, T., Karremans, A. P., Pillco-Huarcaya, R., Azofeifa-Bolaños, J. B., Reubens, B. \& Muys, B. 2020. Vanilla distribution modeling for conservation and sustainable cultivation in a joint land sparing/sharing concept. Agroecosystems 11: e03056. https://doi.org/10.1002/ ecs 2.3056 